\title{
Probing a nonequilibrium Einstein Relation in an aging colloidal glass
}

\author{
Bérengère Abou and François Gallet \\ Laboratoire de Biorhéologie et Hydrodynamique Physico-chimique UMR CNRS \\ 7057 and Fédération de Recherche Matières et Systèmes Complexes FR 2438, \\ 2 Place Jussieu, Case 7056, \\ 75251 Paris Cedex 05, FRANCE
}

(Dated: November 13, 2018)

\begin{abstract}
We present a direct experimental measurement of an effective temperature in a colloidal glass of Laponite, using a micrometric bead as a thermometer. The nonequilibrium fluctuation-dissipation relation, in the particular form of a modified Einstein relation, is investigated with diffusion and mobility measurements of the bead embedded in the glass. We observe an unusual non-monotonic behavior of the effective temperature : starting from the bath temperature, it is found to increase up to a maximum value, and then decreases back, as the system ages. We show that the observed deviation from the Einstein relation is related to the relaxation times previously measured in dynamic light scattering experiments.

PACS numbers: 05.40.-a, 05.20.-y, 05.70.-a
\end{abstract}

At thermodynamical equilibrium, the fluctuationdissipation theorem (FDT) relates the response function of the system to its time autocorrelation function. This theorem may display several forms, depending on the considered observable (Nyquist formula, Einstein relation), but involves a single thermodynamic parameter, which is the equilibrium temperature. However, FDT applies only to ergodic systems at equilibrium, and is not expected to hold for non-equilibrium systems, like glasses and gels, which exhibit relaxation times longer than or comparable to the observation timescale.

Recently, many efforts have been devoted to apply statistical physics concepts to such out-of-equilibrium systems. In particular, fluctuation-dissipation relations have been extended with the help of a timescale dependent effective temperature, different from the bath temperature, and that has been shown to display many of the properties of a thermodynamic temperature [1, 2]. This deviation from equilibrium FDT has been observed in many numerical simulations $1,3,4,45,6,7,8,9,10]$. In these models and simulations, violations are expected to occur when the characteristic observation time $1 / \omega$ is of the same order or greater than the aging time $t_{w}$. On the other hand, there are only few experimental studies of FDT violations in aging materials 11, 12, 13, 14, 15]. In all experimental systems, violations are observed when $1 / \omega$ is smaller than $t_{w}$. This suggests that the aging time $t_{w}$ is not the only relevant parameter to describe aging, as usually considered in numerical simulations, and that microscopic processes, charaterized by the distribution of relaxation times in the system, must be considered.

In this letter we measure the evolution with aging time of an effective temperature $T_{\text {eff }}$ in a colloidal glass of Laponite. This is achieved by simultaneously measuring the mobility - using an optical tweezer - and the position fluctuations of a micrometric bead embedded in the glass. We find that, starting from the bath temperature, $T_{\text {eff }}$ increases with $t_{w}$, up to 1.8 times the bath temperature, and decreases back upon further aging. We propose a consistent interpretation of this unusual behavior by considering the evolution of the relaxation times distribution in the glass.

Let us consider a diffusing particle of mass $m$ evolving in a stationary medium. Its motion is described by a generalized Langevin equation $m d v / d t=F(t)-$ $m \int_{-\infty}^{+\infty} \gamma\left(t-t^{\prime}\right) v\left(t^{\prime}\right) d t^{\prime}$, in which $v(t)=d x / d t$ is the particle velocity, $F(t)$ the random force acting on the particle and $\gamma(t)$ a delayed friction kernel that takes into account the viscoelastic properties of the medium. If the surrounding stationary medium is in thermal equilibrium at temperature $T$, one can derive a generalized Einstein relation, which is a specific form of FDT, $s^{2}\left\langle\Delta \hat{x}^{2}(s)\right\rangle=$ $2 k T \hat{\mu}(s)$, in which $\left\langle\Delta \hat{x}^{2}(s)\right\rangle$ is the mean-square displacement Laplace transform and $\hat{\mu}(s)=1 / m \hat{\gamma}(s)$ the mobility Laplace transform, if inertia is neglected.

The general situation of a particle diffusing in an outof-equilibrium environment is much more difficult to describe. This point has been developed in details in 16] using a generalized Langevin formalism. The FDT is extended with the help of a frequency-dependent effective temperature $T_{\text {eff }}(\omega)$, parametrized by the age $t_{w}$ of the system, and which satisfies [17] :

$$
T_{\text {eff }}(\omega) \Re e \mu(\omega)=\Re e[\mu(\omega) \Theta(\omega)]
$$

where

$$
s^{2}\left\langle\Delta \hat{x}^{2}(s)\right\rangle=2 k \hat{\Theta}(s) \hat{\mu}(s)
$$

The two equations (1) and (2) define an out-ofequilibrium Einstein relation, for a given aging time $t_{w}$. The parameter $\hat{\Theta}(s)$ is related to $\Theta(\omega)=\hat{\Theta}(s=-i \omega)$.

The principle of our experiment to measure the effective temperature in an out-of-equilibrium system is the following : micrometric probes are immersed in a colloidal glass to allow the measurements, at the same aging time $t_{w}$, of both their fluctuating position and mobility. 
$T_{\text {eff }}$ is then obtained from equations (1) and (2). The colloidal glass consists in an aqueous suspension of Laponite $\mathrm{RD}$, a hectorite synthetic clay provided by Laporte Industry. The preparation procedure of the glass has been addressed in details in 18]. These aqueous suspensions form glasses for low volume fraction in particles [19]. Being in a "liquid" state right after preparation, the suspension becomes more and more viscoelastic with time. Since the physical properties of the suspension depend on the time $t_{w}$ elapsed since preparation, the sample is said to age. Aging can be seen through the evolution of both the viscoelastic properties and of the colloidal disks diffusion [18, 20]. This glass, obtained at the ambient temperature, is optically transparent. Moreover, it presents other advantages that make possible to measure an effective temperature with tracer beads. First, the preparation procedure allows to obtain a reproducible initial state, leading to an accurate determination of the origin $t_{w}=0$. Second, the Laponite suspensions age on timescales that depend on the particles concentration. We are thus able to choose the aging timescales of the glass by adjusting this concentration. With a volume fraction of $2.3 \% \mathrm{wt}$, the glass evolves slowly enough to allow two successive measurements - fluctuation and dissipation - without significative aging of the sample. The two measurements are thus performed at the same $t_{w}$.

The experiments were carried out in a square chamber $-20 \times 20 \mathrm{~mm}^{2}$ - made of a microscope plate and a coverslip separated by a thin spacer ( $0.1 \mathrm{~mm}$ thickness). The beads are suspended in the glass right after its preparation. The chamber is then filled with the suspension, sealed with vacuum grease and mounted on a piezoelectric stage on the plate of an optical microscope. The probes are latex and silica beads, in very low concentration (respectively $10^{-4} \%$ and $4.10^{-4} \%$ in volume). Latex beads $(1.0 \pm 0.1 \mu \mathrm{m}$ in diameter, Polysciences, Inc.), were preferentially used for fluctuation measurements : since they do not deposit during the experiment, their random motion is not perturbated by the chamber walls. Silica beads $(2.1 \pm 0.1 \mu \mathrm{m}$ in diameter, Bangs Lab Inc.) were used for dissipation measurements, because they are more efficiently trapped by the optical tweezers. The diameters of the two kinds of probes are close to each other, thus the comparison between the results of the fluctuation and dissipation measurements, once rescaled to the same diameter, is meaningful.

Let us first focus on the two-dimensional brownian motion of a tracer bead immersed in the glass. At a given aging time $t_{w}$, we record the fluctuating motion of a $1 \mu \mathrm{m}$ latex bead during $8 \mathrm{~s}$, with a fast CCD camera sampling at $125 \mathrm{~Hz}$ (Kodak, PS-220). A digital image analysis allows to track the bead positions $x(t)$ and $y(t)$ close to the focus plane of the microscope objective. For each bead, we calculate the time-averaged mean-square displacement $\left\langle\Delta r^{2}(t)\right\rangle_{t^{\prime}}=\left\langle\left(x\left(t^{\prime}+t\right)-x\left(t^{\prime}\right)\right)^{2}+\left(y\left(t^{\prime}+t\right)-\right.\right.$ $\left.\left.y\left(t^{\prime}\right)\right)^{2}\right\rangle_{t^{\prime}}=2\left\langle\Delta x^{2}(t)\right\rangle_{t^{\prime}}$. To preserve a good statistics, we keep the data of $\left\langle\Delta r^{2}(t)\right\rangle_{t^{\prime}}$ in the range $0.008<t<1$ $\mathrm{s}$. The glass remains in a quasi-stationary state during the recording, which takes a short time compared to the aging timescale. The quantity $\left\langle\Delta r^{2}(t)\right\rangle_{t^{\prime}}$ can thus be identified to the ensemble-averaged mean-square displacement $\left\langle\Delta r^{2}(t)\right\rangle_{E}$. Fig. 1 shows the mean-square displacement of latex beads immersed in the colloidal glass, as a function of time $t$, for various aging times $t_{w}$. The mean-square displacement is well described by a power-law behavior $\left\langle\Delta r^{2}(t)\right\rangle_{E}=D t^{\alpha}$ over the full range $0.008<t<1 \mathrm{~s}$. Upon increasing on $t_{w}$, the exponent decreases from $1.05 \pm 0.05$ at the earliest aging times to $0.25 \pm 0.05$ at long aging times. This indicates a nearly diffusive behavior of the tracer bead at short aging times, that becomes sub-diffusive as the glass ages. We describe now the measurement of the mobility $\mu(\omega)$, at a given frequency $\omega$ for various aging times. We recall that this measurement is performed right after the fluctuation motion recording, at the same aging time $t_{w}$. As the Laponite suspension is a viscoelastic fluid, the bead mobility $\mu(\omega)=|\mu(\omega)| e^{i \phi(\omega)}$ is a complex number. We thus need to measure the phase and modulus of the tracer mobility. We use an optical tweezer to trap a $2.1 \mu \mathrm{m}$ silica bead immersed in the glass. Trapping is achieved by focusing a powerful infrared laser beam (Nd YAG, Spectra-Physics, $P_{\max }=600 \mathrm{~mW}$ ) through a microscope objective of large numerical aperture [21]. The trapping force $F$ on a small dielectric object like a silica bead is proportional to the intensity gradient in the focusing region. To first order, one can write $F=-k x$, where $x$ represents the distance of the trapped object from the trap center. The stiffness $k$ is known from an independent calibration. Once the bead is trapped, we make the experimental chamber oscillate by monitoring the displacement $x_{p} \exp (i \omega t)$ of a piezoelectric stage. As a consequence, the viscoelastic glass exerts a sinusodal force $F^{\prime} \exp (i \omega t)$ on the bead. We record with the fast camera the bead movement, and measure by conventional image analysis its displacement $x \exp (i \omega t)$ from the trap center. At a given frequency $\omega$, the force $F^{\prime}(\omega)$ is given

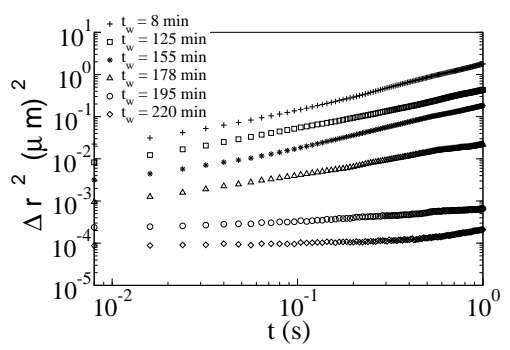

FIG. 1: Mean-square displacement of a $1 \mu \mathrm{m}$ latex bead immersed in the glass, as a function of time. The curves correspond to different $t_{w}=8,125,155,178,195$ and 220 minutes from top to bottom. The fluctuating motion is purely diffusive at short $t_{w}$ and becomes sub-diffusive as the glass ages. 
by $F^{\prime}(\omega)=v(\omega) / \mu(\omega)$, where $v(\omega)=i \omega\left(x_{p}-x\right)$ is the relative glass / bead velocity, and $\mu(\omega)$ the Fourier transform of the bead mobility. In our range of experimental frequencies $(0.5<f<10 \mathrm{~Hz})$, the bead inertia is negligible, so that we can simply use the relation $F+F^{\prime}=0$ to calculate $|\mu(\omega)|$ and $\phi(\omega)$. Notice that the piezoelectric stage and the camera are triggered by two synchronized signals, numerically generated by a PC computer, so that the phase shift between the force and the bead movement can be accurately measured. Fig. 2 (a,b) show the complex mobility of the tracer bead as a function of the aging time $t_{w}$ for various frequencies of the applied force.

Two more steps are necessary to calculate the effective temperature from both fluctuation and mobility data. First, the mean-square displacement is numerically Laplace transformed to the frequency domain $0.15-20$ $\mathrm{Hz}$. In this range, we find that the Laplace transform is well adjusted by a power-law $\left\langle\Delta \hat{r}^{2}(s)\right\rangle=a s^{-b}$. As expected from the observation $\left\langle\Delta r^{2}(t)\right\rangle_{E}=D t^{\alpha}$, the relation $b=\alpha+1$ is accurately verified. Second, we analyze the frequency dependence of the mobility. In the experimental frequency range, $|\mu(\omega)|$ is well fitted by a power law $|\mu(\omega)|=\mu_{0} \omega^{\beta}$, as shown in Fig. 2c. The exponent $\beta$ increases with $t_{w}$ from zero at low aging times to $0.75 \pm 0.05$ at the end of experiment. To

(a)

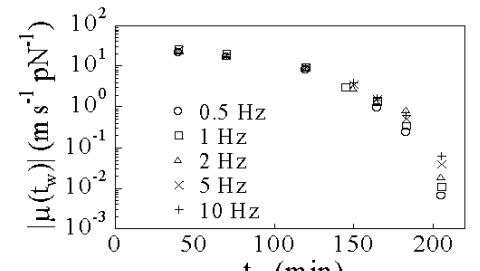

(b)

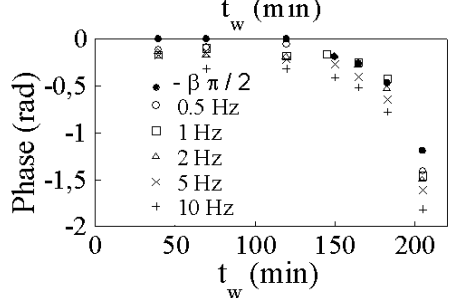

(c)

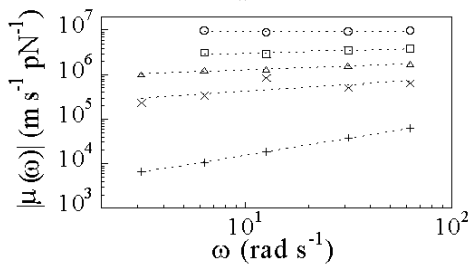

FIG. 2: Modulus (a) and phase (b) of the complex mobility $\mu(\omega)=|\mu(\omega)| e^{i \phi(\omega)}$ of the tracer bead as a function of $t_{w}$, for various frequencies of the applied force. The full circles in (b) correspond to $\phi=-\beta\left(t_{w}\right) \pi / 2$; (c) : mobility modulus versus $\omega$ for various $t_{w}$; from top to bottom, $t_{w}=120,145,165,183,205 \mathrm{~min}$. At low $t_{w},|\mu(\omega)|$ is independent of $\omega$. Upon increasing $t_{w}$, the modulus is well fitted by a power-law $|\mu(\omega)|=\mu_{0} \omega^{\beta}$ with $\beta$ only depending on $t_{w}$. a first-order approximation, we consider that the phase $\phi$ is independent of the frequency. Within a good approximation, its dependence on $t_{w}$ can be related to $\beta$ by $\phi=-\beta\left(t_{w}\right) \pi / 2$ as shown in Fig. 2b. From the analytical form $\mu(\omega)=\mu_{0} \omega^{\beta} \exp (-i \beta \pi / 2)$, the Laplace transform $\hat{\mu}(s)=\mu(\omega=i s)=\mu_{0} s^{\beta}$ is derived by analytical continuation. Using equation (2), we calculate the function $\hat{\Theta}(s)$, parametrized by $t_{w}$, and derive $\Theta(\omega)=\hat{\Theta}(s=-i \omega)$. Finally, the effective temperature, at a given $t_{w}$, is :

$$
k T_{\text {eff }}(\omega)=\frac{a}{4 \mu_{0}} \frac{\cos ((b-2) \pi / 2)}{\cos (\beta \pi / 2)} \omega^{2-b-\beta}
$$

The dependence of $T_{\text {eff }}$ on $t_{w}$ is shown in Fig. 3, at a frequency $f=1 \mathrm{~Hz}$. The results have been averaged over three realizations. At the earliest $t_{w}$, the effective temperature is close to the bath temperature $T_{\text {bath }}=300$ $\mathrm{K}$. Upon increase on $t_{w}, T_{\text {eff }}$ increases up to 1.8 times the bath temperature and then decreases towards $T_{\text {bath }}$ upon further increase on $t_{w}$. This is the first time that such a behavior - increase of $T_{\text {eff followed by a decrease }}$ - is observed in a glassy system. A realistic explanation of this non-monotonic behavior is proposed in the next paragraph. Moreover, our results are in contradiction with electrical measurements of FDT performed in the same system [12], where $T_{\text {eff }}$ is found to decrease with $t_{w}$ and $\omega$ and is larger by about one order of magnitude. A possible origin of this discrepancy may be the choice of different observables in the two experiments [10.

An interpretation of the dependence of $T_{\text {eff }}$ on $t_{w}$ is provided by Dynamic Light Scattering (DLS) and Diffusive Wave Spectroscopy (DWS) experiments, previously performed in colloidal glasses of Laponite 20, 22]. The resulting distribution function of relaxation times $P(\tau)$ is schemed in Fig. 4. Upon increasing $t_{w}$, part of the modes distribution function shift to larger times, while the mode at $\tau \sim 0.1 \mathrm{~ms}$ remains unchanged. When probing our experimental system at a typical frequency $f=1 \mathrm{~Hz}$, three situations occur. At the earliest $t_{w}$, the fast modes $\tau<<1 / f$ allow the system to thermalize with the bath (Fig. 4a). The measured effective temperature is the bath temperature. Upon increase on $t_{w}$, modes $\tau \sim 1 / f$

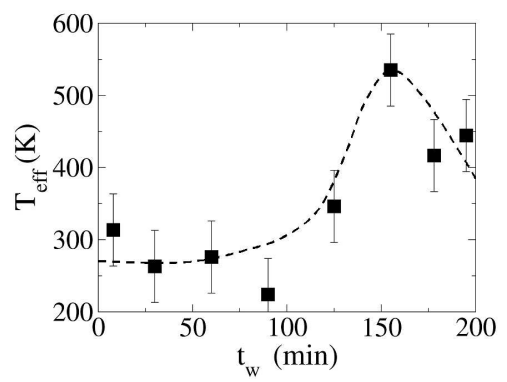

FIG. 3: Effective temperature of the colloidal glass versus $t_{w}$, measured at a frequency $f=1 \mathrm{~Hz}$. Upon increase on $t_{w}, T_{\text {eff }}$ increases up to 1.8 times the bath temperature and decreases back to $T_{\text {bath }}$. The dashed line is a guide for the eyes. 
appear in the system as seen in Fig. 4b. On this observation timescale $1 / f$, the system is out-of-equilibrium : the measured temperature becomes different from the bath temperature and is timescale dependent. Deviation from the equilibrium Einstein relation is thus observed. Finally, for very long $t_{w}$, the fast modes allow the system to thermalize with the bath while the slow ones $\tau \gg 1 / f$ do not play any role at the experimental timescale. $T_{\text {eff }}$ is then expected to reduce back to $T_{\text {bath }}$. These different situations are clearly identified in Fig. 3. However the experimental set-up does not allow to observe a further decrease of $T_{\text {eff }}$ at long $t_{w}$. Indeed, beyond $t_{w}=205 \mathrm{~min}-$ utes, the mobility modulus becomes smaller than $10^{-2} \mathrm{~m}$ $\mathrm{s}^{-1} \mathrm{pN}^{-1}$. In this range, the optical tweezer is not powerful enough to induce a detectable motion of the bead.

In these experiments, deviations are observed when $\omega \tau \sim 1$. This suggests that, besides the waiting time $t_{w}$, the distribution of relaxation times must be included in models to achieve an accurate description of aging.

Let us now comment the dependence of $T_{\text {eff }}$ on the frequency. Since we experimentally find that $2-b-\beta>$

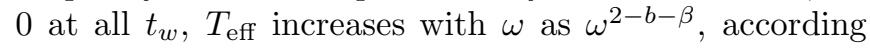
to equation (3). This behavior is consistent with our interpretation at least in the first situation. However, in our picture, one expects $T_{\text {eff }}$ to decrease with $\omega$ at long $t_{w}$. Actually, one can see from Fig. $2 \mathrm{~b}$ that $\phi$ can no longer be accurately considered as independent of $\omega$ at long $t_{w}$. Thus, the analytical interpolation $\mu(\omega)=$ $\mu_{0} \omega^{\beta} \exp -i \beta \pi / 2$ probably breaks down. This difficulty will be soon overcomed by a direct measurement of $\hat{\mu}(s)$ from a creep experiment.

This work provides a test of the nonequilibrium Einstein relation in a colloidal glass, using diffusion and mobility measurements on a micrometric probe. We observe for the first time in a glassy system a non-monotonic behavior of $T_{\text {eff with }} t_{w}$. It seems likely that this behavior is directly related to the evolution of the relaxation times

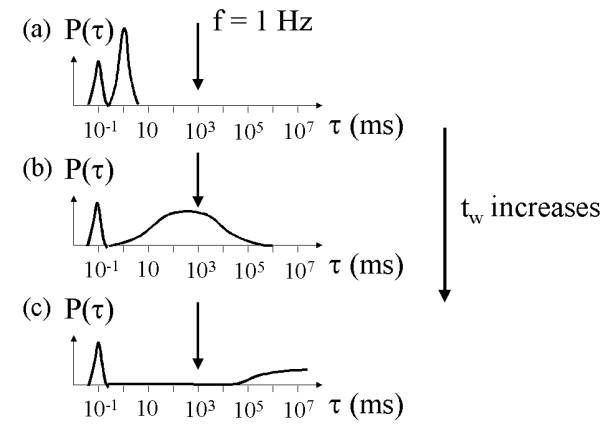

FIG. 4: Scheme of the distribution function of relaxation times $P(\tau)$ in the glass of Laponite (typically $2.5 \%$ wt) at different $t_{w}$. Upon increasing $t_{w}$, part of the modes distribution shifts towards larger times, while the mode at $\tau \sim 0.1 \mathrm{~ms}$ remains unchanged. The arrow represents the measurements timescale $1 / f$. distribution. Notice that deviations from the Einstein relation, found in supercooled liquids even at equilibrium, were explained by spatial heterogeneities [23]. Future works will have to analyze how such heterogeneities interplay with the concept of $T_{\text {eff }}$ - which is an ensembleaveraged quantity. Another open question is whether this effective temperature has a real thermodynamics meaning [2, 10]. The answer will come from experimental tests of FDT involving other physical observables.

We are indebted to N. Pottier for enlightening discussions and comments. We thank P. Monceau for numerical Laplace transformations, M. Balland for help in programmation, and L. Cugliandolo for fruitful exchanges.

[1] Cugliandolo, L., and Kurchan, J. Phys. Rev. Lett. 71, 173 -176 (1993).

[2] Cugliandolo, L.F., Kurchan, J. and Peliti, L. Phys. Rev. E 55, 3898 - 3914 (1997).

[3] Parisi, G. Phys. Rev. Lett. 79, 3660 - 3663 (1997).

[4] Barrat, A. Phys. Rev. E 57, 3629 - 3632 (1998).

[5] Sellitto, M. Eur. Phys. J. B. 4, 135 - 138 (1998).

[6] Marinari, E., Parisi, G., Ricci-Tersenghi, F., and RuizLorenzo, J.J. J. Phys. A : Math Gen. 31, 2611 - 2620 (1998).

[7] Barrat, J.-L., and Kob, W. Europhys. Lett. 46, 637 - 642 (1999).

[8] Berthier, L., Barrat, J.-L., and Kurchan. J. Phys. Rev. E 61, 5464 - 5472 (2000).

[9] Makse, H. A., and Kurchan, J. Nature 415, 614 - 617 (2002).

[10] Fielding, S. and Sollich, P. Phys. Rev. Lett. 88, 050603-1 - 050603-4 (2002).

[11] Grigera, T. S. and Israeloff, N. E. Phys. Rev. Lett. 83, 5038 - 5041 (1999).

[12] Bellon, L., Ciliberto, S. and Laroche, C. Europhys. Lett. 53, 511 - 517 (2001).

[13] Hérisson, D. and Ocio, M. Phys. Rev. Lett. 88, 257202 257205 (2002).

[14] D'Anna, G., Mayor, P., Barrat, A., Loreto, V. and Nori, F. Nature 424, 909 - 912 (2003).

[15] Ojha., R. P., Lemieux, P.-A., Dixon, P. K., Liu, A. J. and Durian, D. J. Nature 427, 521 - 523 (2004).

[16] Pottier, N. Physica A 317, 371 - 1382 (2003); Pottier, N. and Mauger, A. Physica A 332, 15 - 28 (2004).

[17] Pottier, N. cond-mat/0404613 to appear in Physica A.

[18] Abou, B., Bonn, D., and Meunier, J. J. Rheol. 47, 979 988 (2003).

[19] Bonn, D., Tanaka, S., Wegdam, G. H., Kellay, H., and Meunier, J. Europhys. Lett. 45, 52 - 57 1(1999).

[20] Abou, B., Bonn, D., and Meunier, J. Phys. Rev. E 64, 021510 - 021513 (2001).

[21] Hénon, S., Lenormand, G., Richert, A., and Gallet, F. Biophys. J. 76, 1145 - 1151 (1999).

[22] Knaebel A., Bellour M., Munch J.-P., Viasnoff V., Lequeux, F. and Harden, J. L. Europhys. Lett. 52, 73 - 79 (2000).

[23] Swallen, S. F., Bonvallet, P. A., McMahon, R. J., and Ediger, M. D. Phys. Rev. Lett. 90, 015901 1-4 (2003). 